\title{
ON THE CUBIC MOMENT OF QUADRATIC DIRICHLET $L$-FUNCTIONS
}

\author{
QIAO ZHANG
}

\section{Introduction}

An important problem in analytic number theory is to understand the $m$-th moment of quadratic Dirichlet $L$-functions

$$
\sum_{\substack{|D| \leq x \\ \text { fund. disc. }}} L\left(\frac{1}{2}, \chi_{D}\right)^{m}
$$

where $\chi_{D}$ is the quadratic Dirichlet character associated to $\mathbb{Q}(\sqrt{D})$ as defined in [11]. Besides its own interest, this mean value problem also plays a crucial role in the studies of such as the Lindelöf Conjecture and the folklore non-vanishing conjecture that $L\left(\frac{1}{2}, \chi_{D}\right) \neq 0$. See, for example, [5] [7] [12].

Jutila [7] was the first to obtain the asymptotic formulas for the cases $m=1,2$, and Soundararajan [12] succeeded in the cubic case. For higher moments, a good upper bound has been obtained by Heath-Brown [6] in the quartic case, but their asymptotic formulas are still out of reach. In general, motivated by the fundamental work of Katz and Sarnak [8] on symmetric types associated to families of $L$-functions, and by calculations of Keating and Snaith [9] based on random matrix theory, Conrey and Farmer have made the following conjecture

$$
\sum_{\substack{|D| \leq x \\ D \text { fund. disc. }}} L\left(\frac{1}{2}, \chi_{D}\right)^{m} \sim x R_{m}(\log x)
$$

for some polynomial $R_{m}$ of degree $\frac{m(m+1)}{2}$; see also [2]. Many even suggest that the error term here be of order $x^{\frac{1}{2}+\epsilon}$.

Diaconu, Goldfeld and Hoffstein [4] studied this problem through the approach of multiple Dirichlet series and gave another heuristic argument of (1.2). In particular, to analyze the cubic moment, they considered the multiple Dirichlet series

$$
Z(s, w)=\sum_{D \text { fund. disc. }} \frac{L\left(s, \chi_{D}\right)^{3}}{|D|^{w}},
$$

Received November 17, 2003. 
and its related functions (for notations, see Section 2)

$$
\begin{aligned}
& Z(s, w ; a, b)=\sum_{\substack{d=1 \\
\text { odd sq. free }}}^{\infty} \frac{L^{2}\left(s, \chi_{d} \chi_{a}\right)^{3} \chi_{b}(d)}{d^{w}}, \\
& Z(s, w ; a, b ; r)=\sum_{\substack{d=1 \\
r^{2} \mid d, 2 \nmid d}}^{\infty} \frac{L^{2}\left(s, \chi_{a} \chi_{d}\right)^{3} P_{d}^{a}(s)}{d^{w}},
\end{aligned}
$$

where $a, b \in \mathbb{Z}$ with $a b= \pm 1, \pm 2$ and $P_{d}^{a}(s)$ is a Dirichlet polynomial as defined in Section 3. For $s$ in a neighborhood of $\frac{1}{2}$, the authors showed that $Z(s, w ; a, b ; r)$ can be meromorphically continued up to $\Re w>0$ and that the series expansion

$$
Z(s, w ; a, b)=\sum_{\substack{r=1 \\ 2 \nmid r}}^{\infty} \mu(r) Z(s, w ; a, b ; r)
$$

converges absolutely for $\Re w>\frac{4}{5}$, thus obtaining the meromorphic continuation of $Z(s, w ; a, b)$, as well as $Z(s, w)$ (c.f. (4.2)), in this region. With this information, the authors were able to establish the asymptotic formula for the cubic moment of quadratic Dirichlet $L$-functions with the best known error term

$$
\sum_{|D| \leq x} L\left(\frac{1}{2}, \chi_{D}\right)^{3}=x R_{3}(\log x)+O\left(x^{\frac{47-\sqrt{265}}{36}+\epsilon}\right) .
$$

The present paper continues the study of analytic properties for $Z(s, w)$. Under certain technical assumptions, we find that $Z\left(\frac{1}{2}, w\right)$ has a simple pole at $w=\frac{3}{4}$ and its residue is explicitly computed. More precisely, we have the following result.

Theorem 1. Let $\sigma_{0} \in\left(\frac{1}{2}, \frac{3}{4}\right)$ be a constant. Assume that, for $s$ in a neighborhood of $\frac{1}{2}$, every $Z(s, w ; a, b)$ has a meromorphic continuation to $\Re w>\sigma_{0}$, and that the series expansion (1.6) is valid in this region. Then $Z\left(\frac{1}{2}, w\right)$ can be analytically continued up to $\Re w>\sigma_{0}$ and has a simple pole at $w=\frac{3}{4}$ with residue

$$
\operatorname{Res}_{w=\frac{3}{4}} Z\left(\frac{1}{2}, w\right)=\frac{223-253 \sqrt{2}}{256} P\left(\frac{\Gamma_{\mathbb{R}}\left(\frac{1}{4}\right)^{4}}{\Gamma_{\mathbb{R}}\left(\frac{3}{4}\right)^{4}}+\frac{\Gamma_{\mathbb{R}}\left(\frac{1}{4}\right) \Gamma_{\mathbb{R}}\left(\frac{5}{4}\right)^{3}}{\Gamma_{\mathbb{R}}\left(\frac{3}{4}\right) \Gamma_{\mathbb{R}}\left(\frac{7}{4}\right)^{3}}\right) \approx-0.162,
$$

where

$$
\begin{aligned}
P & =\frac{\zeta\left(\frac{1}{2}\right)^{7}}{\zeta_{2}\left(\frac{1}{2}\right)^{3}} \prod_{p>2}\left(1-\frac{14}{p^{\frac{3}{2}}}-\frac{1}{p^{2}}+\frac{78}{p^{\frac{5}{2}}}-\frac{84}{p^{3}}-\frac{58}{p^{\frac{7}{2}}}+\frac{154}{p^{4}}-\frac{70}{p^{\frac{9}{2}}}-\frac{49}{p^{5}}+\frac{64}{p^{\frac{11}{2}}}-\frac{22}{p^{6}}+\frac{1}{p^{7}}\right) \\
& \approx-0.00193 .
\end{aligned}
$$

The existence of the pole of $Z\left(\frac{1}{2}, w\right)$ at $w=\frac{3}{4}$, together with some suitable growth conditions for $Z\left(\frac{1}{2}, w\right)$ itself, would give us information about the cubic moment of quadratic Dirichlet $L$-functions. For example, we have 
Theorem 2. Assume that the conditions in Theorem 1 are satisfied, and that

$$
Z\left(\frac{1}{2}, \sigma_{0}+i t\right) \ll(2+|t|)^{r+\epsilon}
$$

for some positive constant $r<3-4 \sigma_{0}$. Then we have

$$
\sum_{\substack{|D| \leq x \\ \text { fund. disc. }}} L\left(\frac{1}{2}, \chi_{D}\right)^{3}=x R_{3}(\log x)+b x^{\frac{3}{4}}+O\left(x^{\frac{r+\sigma_{0}}{r+1}+\epsilon}\right),
$$

where $b \approx-0.215$ is a constant.

Since our main interest is to study the existence of the possible "exceptional main term" $x^{\frac{3}{4}}$, in Theorem 2 we do not seek the weakest possible growth condition for (1.11). A less restrictive condition on $r$ would, at least, lead to a similar asymptotic expansion as in (1.11) for certain weighted cubic moments. One may observe, from Propositions 1 and 2, that $Z\left(\frac{1}{2}, w\right)$ (or more precisely, the function $Z_{M}\left(\frac{1}{2}, w ; a, b\right)$ as defined in (3.4)) satisfies certain functional equation of form $w \mapsto 1-w$, so a application of the Phragmen-Lindelöf Principle suggests that we might be able to take $r=\epsilon$. Such an estimate is still beyond our reach, but in [4] it is shown that we can take $r=5(1-\sigma)$ for $\sigma>\frac{4}{5}$, which is already sufficient to yield a weighted cubic moment with error terms of order $x^{\frac{4}{5}+\epsilon}$.

The asymptotic expression (1.11) can be compared with the available data [10] for $x \leq 10^{7}$, and numerical calculations over a much larger region are expected. The appearance of the exceptional main term $x^{\frac{3}{4}}$ suggests an unexpectedly fine structure for moments of quadratic Dirichlet $L$-functions (1.1). It will be interesting to locate such exceptional main terms for higher moments, and we will return to this topic in a separate paper.

The paper is organized as follows. In Section 2 we set the notations. Section 3 introduces a family of multiple Dirichlet series $Z_{M}(s, w ; a, b)$ as well as their analytic properties, especially their functional equations. These functional equations readily imply the appearance of the pole for $Z\left(\frac{1}{2}, w\right)$ at $w=\frac{3}{4}$; c.f. the remarks following Proposition 2. In Section 4, the functional equations are applied to compute the residues of $Z_{M}(s, w ; a, b)$ along some polar lines, which in turn leads to the determination of the residue of $Z\left(\frac{1}{2}, w\right)$ at $w=\frac{3}{4}$. This proves Theorem 1. Finally, Theorem 2 is proved in Section 5.

\section{Symbols and Notations}

For a (positive or negative) integer $\ell$, we always write $\ell=\ell_{0} \ell_{1}^{2}$, where $\ell_{0}$ is

squarefree and $\ell_{1}$ is positive. Also, we write $\delta_{\ell}=\operatorname{sgn}(\ell)$, and let $\widetilde{\chi}_{\ell_{0}}=\left(\dot{\dot{\ell_{0}}}\right)$ be the quadratic Dirichlet character of conductor $\ell_{0}$. Furthermore, we introduce some variants of the divisor function.

$$
\tau_{3}(m)=\sum_{m_{1} m_{2} m_{3}=m} 1, \quad \tau_{3}(m ; L)=\sum_{\substack{m_{1} m_{2} m_{3}=m \\ m_{1}, m_{2}, m_{3} \mid L}} 1,
$$




$$
\tau_{3}(m ; L ; \mu)=\sum_{\substack{m_{1} m_{2} m_{3}=m \\ m_{1}, m_{2}, m_{3} \mid L}} \mu\left(m_{1}\right) \mu\left(m_{2}\right) \mu\left(m_{3}\right) .
$$

For every integer $N \geq 1$, we let

$$
\zeta_{N}(s)=\prod_{p \mid N}\left(1-\frac{1}{p^{s}}\right)^{-1}, \quad \zeta^{N}(s)=\frac{\zeta(s)}{\zeta_{N}(s)}
$$

denote the local and partial Riemann zeta functions at $N$ respectively. Similarly, for every Dirichlet character $\chi$ we also write

$$
L_{N}(s, \chi)=\prod_{p \mid N}\left(1-\frac{\chi(p)}{p^{s}}\right)^{-1}, \quad L^{N}(s, \chi)=\frac{L(s, \chi)}{L_{N}(s, \chi)} .
$$

For $\epsilon= \pm 1$, we write

$$
\Gamma_{\mathbb{R}}(s)=\pi^{-\frac{s}{2}} \Gamma\left(\frac{s}{2}\right), \quad \Gamma_{\epsilon}(s)= \begin{cases}\Gamma_{\mathbb{R}}(s), & \text { if } \epsilon=1 \\ \Gamma_{\mathbb{R}}(s+1), & \text { if } \epsilon=-1\end{cases}
$$

Finally, for reference we list some functions that will be used extensively in our future discussions.

$$
B_{m}(s)=\prod_{p \mid m}\left(1+\frac{3}{p^{2-2 s}}-\frac{9}{p}-\frac{3}{p^{3-2 s}}+\frac{3}{p^{2 s}}+\frac{9}{p^{2}}-\frac{3}{p^{1+2 s}}-\frac{1}{p^{3}}\right),
$$

$$
\begin{aligned}
& B_{m}^{*}(s)=\prod_{p \mid m}\left(\frac{3}{p^{1-s}}+\frac{1}{p^{3-3 s}}-\frac{3}{p^{s}}-\frac{9}{p^{2-s}}+\frac{9}{p^{1+s}}+\frac{3}{p^{3-s}}-\frac{1}{p^{3 s}}-\frac{3}{p^{2+s}}\right), \\
& A_{m}(s, \chi)=\prod_{p \mid m}\left(1-\frac{\chi(p)^{2}}{p}+\left(p^{\frac{1-3 s}{2}}-p^{\frac{3 s-3}{2}}\right) \frac{\chi(p) p^{\frac{3 s-3}{2}}}{\zeta_{p}(s)^{3} B_{p}\left(\frac{2-s}{2}\right)}\right),
\end{aligned}
$$

$$
A_{m}^{*}(s, \chi)=\prod_{p \mid m}\left(\chi(p)\left(p^{\frac{1-3 s}{2}}-p^{\frac{3 s-3}{2}}\right)+\left(1-\frac{\chi(p)^{2}}{p}\right) \frac{p^{\frac{3 s-3}{2}}}{\zeta_{p}(s)^{3} B_{p}\left(\frac{2-s}{2}\right)}\right),
$$

$$
\begin{gathered}
A_{N}(s, \chi ; a)=A_{\frac{N}{a}}(s, \chi) A_{a}^{*}(s, \chi), \quad E_{N}(s ; b)=B_{\frac{N}{b}}(s) B_{b}^{*}(s) \\
R_{M}(s)=\frac{\zeta(2 s)^{6} \zeta(6 s-1)}{\zeta_{M}(1) \zeta_{M}(2 s)^{3} \zeta_{M}(6 s-1)}
\end{gathered}
$$




\section{Functional Equations}

Let $M$ be a positive squarefree even integer and $a, b$ its (positive or negative) coprime divisors, then [1] shows that, for every integers $d, n$ coprime to $N$, there exist (unique) Dirichlet polynomials $P_{d}^{a}(s), Q_{n}^{b}(w)$ such that

$$
\sum_{\substack{d=1 \\(d, M)=1}}^{\infty} \frac{L^{M}\left(s, \chi_{d_{0}} \chi_{a}\right)^{3} \chi_{b}\left(d_{0}\right) P_{d}^{a}(s)}{d^{w}}=\sum_{\substack{n=1 \\(n, M)=1}}^{\infty} \frac{L^{M}\left(w, \tilde{\chi}_{n_{0}} \chi_{b}\right) \chi_{a}\left(n_{0}\right) Q_{n}^{b}(w)}{n^{s}}
$$

Now define the multiple Dirichlet series

$$
Z_{M}(s, w ; a, b)=\sum_{\substack{d=1 \\(d, M)=1}}^{\infty} \frac{L^{M}\left(s, \chi_{d_{0}} \chi_{a}\right)^{3} \chi_{b}\left(d_{0}\right) P_{d}^{a}(s)}{d^{w}},
$$

then it is known [1] [4] that $Z_{M}(s, w ; a, b)$ has a meromorphic continuation in $s, w$ to everywhere, and that the only possible poles for $Z_{M}\left(\frac{1}{2}, w ; a, b\right)$ are at $w=0,1, \frac{3}{4}$. Furthermore, $w=1$ is a pole for $Z_{M}(s, w ; a, b)$ only if $b=1$, and this pole, if it exists, must be simple with residue

$$
\operatorname{Res}_{w=1} Z_{M}(s, w ; a, 1)=\frac{R_{M}(s)}{\zeta_{M}(2 s)^{3}}=\frac{\zeta(2 s)^{6} \zeta(6 s-1)}{\zeta_{M}(1) \zeta_{M}(2 s)^{6} \zeta_{M}(6 s-1)},
$$

where $R_{M}(s)$ is as defined in (2.11). The possible pole $w=\frac{3}{4}$, which must be simple if it exists, comes from the polar line $w=\frac{3-3 s}{2}$ for $Z_{M}(s, w ; a, b)$.

The basic properties of $Z_{M}(s, w ; a, b)$ are the following functional equations, one coming from those of quadratic Dirichlet $L$-functions $L\left(s, \chi_{d_{0}} \chi_{a}\right)^{3}$ and (3.2), and the other coming from the "quadratic reciprocity law" (3.3). They were indicated in [1] [4], but for our purposes we need their exact formulations, so we list them explicitly as follows. The proofs are essentially the same as those for Propositions 4.2 and 4.3 in [4], and we omit them here.

Proposition 1. The function $Z_{M}(s, w ; a, b)$ satisfies a functional equation

$$
(s, w) \mapsto\left(1-s, w+3 s-\frac{3}{2}\right) ;
$$

more precisely, we have

$$
\begin{aligned}
Z_{M}(s, w ; a, b) & =\frac{\zeta_{\frac{M}{a}}(2-2 s)^{3}}{2|a|^{3 s-\frac{3}{2}}} \frac{\Gamma_{\delta_{a}}(1-s)^{3}}{\Gamma_{\delta_{a}}(s)^{3}} \sum_{u, v \mid\left(\frac{M}{a}\right)^{3}} \frac{\tau_{3}\left(u ; \frac{M}{a} ; \mu\right) \tau_{3}\left(v ; \frac{M}{a}\right)}{u^{s} v^{1-s}} \\
& \times \sum_{\epsilon= \pm 1} D_{\epsilon}(s ; a, u, v) Z_{M}\left(1-s, w+3 s-\frac{3}{2} ; a, \epsilon(b u v)_{0}\right)
\end{aligned}
$$


where

$$
D_{\epsilon}(s ; a, u, v)= \begin{cases}\chi_{a}(u v)\left(1+\epsilon \frac{2^{3-6 s} \chi_{-1}(u v)}{\zeta_{2}(2-2 s)^{3}}\right), & \text { if } a \equiv 1(\bmod 4) \\ 2^{3-6 s} \chi_{a}(u v)\left(1+\epsilon \cdot \chi_{-1}(u v)\right), & \text { if } a \equiv 2(\bmod 4) \\ \chi_{(u v)_{0}}(a)\left(\frac{2^{3-6 s} \chi_{-1}(u v)}{\zeta_{2}(2-2 s)^{3}}+\epsilon\right), & \text { if } a \equiv-1(\bmod 4) .\end{cases}
$$

Proposition 2. The function $Z_{M}(s, w ; a, b)$ satisfies a functional equation

$$
(s, w) \mapsto\left(s+w-\frac{1}{2}, 1-w\right) ;
$$

more precisely, we have

$$
\begin{aligned}
& Z_{M}(s, w ; a, b)=\frac{\zeta_{\frac{M}{D_{b}}}(2-2 w) D_{b}(w)}{2|b|^{w-\frac{1}{2}}} \sum_{u, v \mid \frac{M}{D_{b^{b}}}} \frac{\mu(u) \chi_{b}(u v)}{u^{w} v^{1-w}} \sum_{\epsilon= \pm 1} \frac{\Gamma_{\delta_{b} \epsilon}(1-w)}{\Gamma_{\delta_{b} \epsilon}(w)} \\
& \times\left(Z_{M}\left(s+w-\frac{1}{2}, 1-w ;(a u v)_{0}, b\right)+\epsilon \cdot Z_{M}\left(s+w-\frac{1}{2}, 1-w ;-(a u v)_{0}, b\right)\right),
\end{aligned}
$$

where

$$
D_{b}(w)=\left\{\begin{array}{ll}
1, & \text { if } b \equiv 1(\bmod 4) ; \\
2^{1-2 w}, & \text { if } b \neq \equiv 1(\bmod 4),
\end{array} \quad D_{b}= \begin{cases}2, & \text { if } b \equiv-1(\bmod 4) ; \\
1, & \text { if } b \neq \equiv-1(\bmod 4) .\end{cases}\right.
$$

One may observe that these functional equations imply that $Z_{M}\left(\frac{1}{2}, w ; a, b\right)$ has a possible simple pole at $w=\frac{3}{4}$. In fact, as shown in [4], $Z_{M}(s, w ; a, b)$ can be analytically continued to everywhere through these functional equations, so its poles can only come from those transformed during this process from the original polar line $w=1$. Now (3.5) shows that $Z_{M}(s, w ; a, b)$ has a simple pole at $(s, w)=\left(\frac{1}{4}, 1\right)$. The first functional equation (3.6) transforms this simple pole to $(s, w)=\left(\frac{3}{4}, \frac{1}{4}\right)$, and then the second functional equation (3.7) takes it to $(s, w)=\left(\frac{1}{2}, \frac{3}{4}\right)$. It is easy to check that this is the only origin for the possible pole at $w=\frac{3}{4}$ for $Z_{M}\left(\frac{1}{2}, w ; a, b\right)$, so the only question that remains is whether $w=\frac{3}{4}$ is indeed a pole, i.e., whether the residue of $Z_{M}\left(\frac{1}{2}, w ; a, b\right)$ at $w=\frac{3}{4}$ is nonzero.

\section{Computation of Residues}

In this section, we apply the above functional equations to compute the residue of $Z_{M}(s, w ; a, b)$ at $w=\frac{3-3 s}{2}$; in particular, this gives the residue of $Z_{M}\left(\frac{1}{2}, w ; a, b\right)$, and so that of $Z\left(\frac{1}{2}, w\right)$, at $w=\frac{3}{4}$.

To begin with, we apply (3.5) to Proposition 1.

Proposition 3. We have

$$
\operatorname{Res}_{w=\frac{5}{2}-3 s} Z_{M}(s, w ; a, b)=\frac{1}{2|a|^{3 s-\frac{3}{2}}} \frac{\Gamma_{\delta_{a}}(1-s)^{3}}{\Gamma_{\delta_{a}}(s)^{3}} \frac{R_{M}(1-s) E_{\frac{M}{a}}(s ; b) D_{b}(s ; a)}{\zeta_{a}(2-2 s)^{3}},
$$


where $R_{M}(s)$ is as defined in (2.11) and

$$
D_{b}(s ; a)= \begin{cases}\chi_{a}(|b|)\left(1+\delta_{b} \frac{2^{3-6 s} \chi_{-1}(|b|)}{B_{2}(s) \zeta_{2}(2-2 s)^{3}}\right), & \text { if } a \equiv 1(\bmod 4) ; \\ 2^{3-6 s} \chi_{a}(|b|)\left(1+\delta_{b} \chi_{-1}(|b|)\right), & \text { if } a \equiv 2(\bmod 4) ; \\ \chi_{a}(|b|)\left(\delta_{b} \chi_{-1}(|b|)+\frac{2^{3-6 s}}{B_{2}(s) \zeta_{2}(2-2 s)^{3}}\right), & \text { if } a \equiv-1(\bmod 4), 2 \nmid b ; \\ \delta_{b} \chi_{-a}\left(\frac{|b|}{2}\right) \chi_{2}(a), & \text { if } a \equiv-1(\bmod 4), 2 \mid b .\end{cases}
$$

Proof. First assume that $a \equiv-1(\bmod 4)$, then $(3.6)$ gives

$$
\begin{aligned}
& Z_{M}(s, w ; a, b)=\frac{\zeta_{\frac{M}{a}}(2-2 s)^{3}}{2|a|^{3 s-\frac{3}{2}}} \frac{\Gamma_{\delta_{a}}(1-s)^{3}}{\Gamma_{\delta_{a}}(s)^{3}} \sum_{u, v \mid\left(\frac{M}{2 a}\right)^{3}} \frac{\tau_{3}\left(u ; \frac{M}{2 a} ; \mu\right) \tau_{3}\left(v ; \frac{M}{2 a}\right) \chi_{(u v)_{0}}(a)}{u^{s} v^{1-s}} \\
& \quad \times\left(\sum_{\epsilon= \pm 1}\left(\frac{2^{3-6 s} \chi_{-1}(u v)}{\zeta_{2}(2-2 s)^{3}}+\epsilon B_{2}(s)\right) Z_{M}\left(1-s, w+3 s-\frac{3}{2} ; a, \epsilon(b u v)_{0}\right)\right. \\
& \left.\quad+\chi_{2}(a) B_{2}^{*}(s) \sum_{\epsilon= \pm 1} \epsilon \cdot Z_{M}\left(1-s, w+3 s-\frac{3}{2} ; a, 2 \epsilon(b u v)_{0}\right)\right) .
\end{aligned}
$$

If $2 \nmid b$, then we have

$$
\begin{aligned}
\underset{\substack{w=\frac{5}{2}-3 s \\
\operatorname{Res}}}{ } Z_{M}(s, w ; a, b)=\frac{\chi_{a}(|b|)}{2|a|^{3 s-\frac{3}{2}}} \frac{\Gamma_{\delta_{a}}(1-s)^{3}}{\Gamma_{\delta_{a}}(s)^{3}} \frac{R_{M}(1-s)}{\zeta_{a}(2-2 s)^{3}}\left(\frac{2^{3-6 s}}{\zeta_{2}(2-2 s)^{3}}+\delta_{b} \chi_{-1}(|b|) B_{2}(s)\right) \\
\times \sum_{\substack{u, v\left|\left(\frac{M}{2 a}\right)^{3}\\
\right| b u v \mid=\square}} \frac{\tau_{3}\left(u ; \frac{M}{2 a} ; \mu\right) \tau_{3}\left(v ; \frac{M}{2 a}\right)}{u^{s} v^{1-s}} \\
=\frac{\chi_{a}(|b|)}{2|a|^{3 s-\frac{3}{2}}} \frac{\Gamma_{\delta_{a}}(1-s)^{3}}{\Gamma_{\delta_{a}}(s)^{3}} \frac{R_{M}(1-s) E_{\frac{M}{2 a}}(s ; b)}{\zeta_{a}(2-2 s)^{3}}\left(\frac{2^{3-6 s}}{\zeta_{2}(2-2 s)^{3}}+\delta_{b} \chi_{-1}(|b|) B_{2}(s)\right) \\
=\frac{\chi_{a}(|b|)}{2|a|^{3 s-\frac{3}{2}}} \frac{\Gamma_{\delta_{a}}(1-s)^{3}}{\Gamma_{\delta_{a}}(s)^{3}} \frac{R_{M}(1-s) E_{\frac{M}{a}}(s ; b)}{\zeta_{a}(2-2 s)^{3}}\left(\frac{2^{3-6 s}}{\zeta_{2}(2-2 s)^{3} B_{2}(s)}+\delta_{b} \chi_{-1}(|b|)\right) .
\end{aligned}
$$

This proves the proposition in the present case. The other cases can be studied similarly, so we omit the details.

To determine the residues along the polar line $w=\frac{3-3 s}{2}$, we apply Proposition 2 upon Proposition 3.

Proposition 4. We have

$$
\operatorname{Res}_{w=\frac{3-3 s}{2}} Z_{M}(s, w ; a, b)=\frac{\zeta_{\frac{M}{b}}(3 s-1)}{8|b|^{\frac{2-3 s}{2}}} R_{M}\left(\frac{s}{2}\right) E_{M}\left(\frac{2-s}{2} ; b\right) C(s ; a, b),
$$

where, for $\delta, \epsilon= \pm 1$, we write

$$
\Gamma_{\delta}^{\epsilon}(s)=\frac{\Gamma_{\delta \epsilon}\left(\frac{s}{2}\right)^{3}}{\Gamma_{\delta \epsilon}\left(\frac{2-s}{2}\right)^{3}}\left(\frac{\Gamma_{+}\left(\frac{3 s-1}{2}\right)}{\Gamma_{+}\left(\frac{3-3 s}{2}\right)}+\epsilon \frac{\Gamma_{-}\left(\frac{3 s-1}{2}\right)}{\Gamma_{-}\left(\frac{3-3 s}{2}\right)}\right), \quad G_{\delta}(s)=\Gamma_{\delta}^{+}(s)+\Gamma_{\delta}^{-}(s)
$$


and $C(s ; a, b)$ equals to

$$
\begin{cases}\chi_{a}(|b|) A_{\frac{M}{2 b}}(s, 1 ; a) G_{\delta_{a}}(s)\left(\frac{2^{3 s-3}+2^{3 s-4}-2^{6 s-5}}{\zeta_{2}(s)^{3} B_{2}\left(1-\frac{s}{2}\right)}+\frac{1}{2}\right), & \text { if } b \equiv 1(\bmod 4), 2 \nmid a ; \\ \chi_{a}(|b|) A_{\frac{M}{2 b}}\left(s, 1 ; \frac{a}{2}\right) G_{\delta_{a}}(s)\left(2^{\frac{1-3 s}{2}}-2^{\frac{3 s-3}{2}}+\frac{2^{\frac{3 s-5}{2}}}{B_{2}\left(1-\frac{s}{2}\right) \zeta_{2}(s)^{3}}\right) & \text { if } b \equiv 1(\bmod 4), 2 \mid a ; \\ \frac{\chi_{b}(|a|)}{2^{3-3 s}} G_{\delta_{a}}(s) \sum_{\epsilon= \pm 1} \chi_{\epsilon}(a)\left(1+\epsilon \delta_{b}\right) A_{\frac{M}{b}}\left(s, \chi_{\epsilon} ; a\right), & \text { if } b \equiv 2(\bmod 4) ; \\ \frac{\chi_{a}(|b|) \chi_{-1}(a) G_{\delta_{a}}(s) A_{\frac{M}{2 b}}(s, 1 ; a)}{2^{2-3 s} \zeta_{2}(3 s-1)}\left(1-\frac{2^{3 s-3}}{\zeta_{2}(s)^{3} B_{2}\left(1-\frac{s}{2}\right)}\right), & \text { if } b \equiv-1(\bmod 4) .\end{cases}
$$

Proof. If $b \equiv 1(\bmod 4)$, we may check that $\chi_{(a u v)_{0}}(|b|) \chi_{b}(u v)=\chi_{a}(|b|)$ and $\chi_{-1}(|b|)=\delta_{b}$, so combining Propositions 2 and 3 we have

$$
\begin{aligned}
& \underset{\substack{\text { Res } \\
w=\frac{3-3 s}{2}}}{ } Z_{M}(s, w ; a, b) \\
& =\frac{\zeta_{\frac{M}{b}}(3 s-1)}{8|b|^{\frac{2-3 s}{2}}} \sum_{u, v \mid \frac{M}{b}} \frac{\mu(u) \chi_{b}(u v)}{u^{\frac{3-3 s}{2}} v^{\frac{3 s-1}{2}}} \frac{R_{M}\left(\frac{s}{2}\right) E_{\frac{M}{(a u v)_{0}}}\left(1-\frac{s}{2} ; b\right)}{\left|(a u v)_{0}\right|^{\frac{3-3 s}{2}} \zeta_{(a u v)_{0}}(s)^{3}} \sum_{\epsilon= \pm 1} \frac{\Gamma_{\delta_{b} \epsilon}\left(\frac{3 s-1}{2}\right)}{\Gamma_{\delta_{b} \epsilon}\left(\frac{3-3 s}{2}\right)} \\
& \times\left(\frac{\Gamma_{\delta_{a}}\left(\frac{s}{2}\right)^{3} D_{b}\left(1-\frac{s}{2} ;(a u v)_{0}\right)}{\Gamma_{\delta_{a}}\left(1-\frac{s}{2}\right)^{3}}+\epsilon \frac{\Gamma_{-\delta_{a}}\left(\frac{s}{2}\right)^{3} D_{b}\left(1-\frac{s}{2} ;-(a u v)_{0}\right)}{\Gamma_{-\delta_{a}}\left(1-\frac{s}{2}\right)^{3}}\right)
\end{aligned}
$$

whence in $(4.1)$ we have

$$
\begin{aligned}
C(s ; a, b) & =\sum_{u, v \mid \frac{M}{b}} \frac{\mu(u) \chi_{b}(u v)}{u^{\frac{3-3 s}{2}} v^{\frac{3 s-1}{2}}} \frac{\left|(a u v)_{0}\right|^{\frac{3 s-3}{2}}}{B_{(a u v)_{0}}\left(1-\frac{s}{2}\right) \zeta_{(a u v)_{0}}(s)^{3}} \\
& \times\left(\Gamma_{\delta_{a}}^{+}(s) D_{b}\left(1-\frac{s}{2} ;(a u v)_{0}\right)+\delta_{b} \Gamma_{\delta_{a}}^{-}(s) D_{b}\left(1-\frac{s}{2} ;-(a u v)_{0}\right)\right) .
\end{aligned}
$$

In case $2 \nmid a$, this gives

$$
\begin{aligned}
& C(s ; a, b)=\sum_{\substack{u, v\left|\frac{M}{b} \\
2\right|(a u v)_{0}}}+\sum_{\substack{u, v \mid \frac{M}{b} \\
2 \nmid(a u v)_{0}}}=\sum_{\substack{u, v \mid \frac{M}{b} \\
2 \| u v}}+\frac{1}{2} \sum_{u, v \mid \frac{M}{2 b}} \\
& \quad=\sum_{u, v \mid \frac{M}{2 b}} \frac{\chi_{a}(|b|) \mu(u)\left|(a u v)_{0}\right|^{\frac{3 s-3}{2}} G_{\delta_{a}}(s)}{u^{\frac{3-3 s}{2}} v^{\frac{3 s-1}{2}} B_{(a u v)_{0}}\left(1-\frac{s}{2}\right) \zeta_{(a u v)_{0}}(s)^{3}}\left(\frac{2^{3 s-3}+2^{3 s-4}-2^{6 s-5}}{\zeta_{2}(s)^{3} B_{2}\left(1-\frac{s}{2}\right)}+\frac{1}{2}\right) \\
& =\chi_{a}(|b|) A_{\frac{M}{2 b}}(s, 1 ; a) G_{\delta_{a}}(s)\left(\frac{2^{3 s-3}+2^{3 s-4}-2^{6 s-5}}{\zeta_{2}(s)^{3} B_{2}\left(1-\frac{s}{2}\right)}+\frac{1}{2}\right) .
\end{aligned}
$$

Hence the proposition is proved in the case that $b \equiv 1(\bmod 4)$ and $2 \nmid a$. The other cases can be proved similarly.

To compute the residue of $Z\left(\frac{1}{2}, w\right)$ at $w=\frac{3}{4}$, the last ingredient is the relation between $Z_{M}(s, w ; a, b)$ and $Z(s, w)$. 
Proposition 5. Let $Z(s, w ; a, b)$ be as defined in (1.4). Then we have

$$
\begin{aligned}
& Z(s, w)=\frac{\left(1-\frac{1}{2^{s}}\right)^{-3}+\left(1+\frac{1}{2^{s}}\right)^{-3}+2^{1-2 w}}{4}(Z(s, w ; 1,1)+Z(s, w ;-1,1)) \\
& \quad+\frac{\left(1-\frac{1}{2^{s}}\right)^{-3}+\left(1+\frac{1}{2^{s}}\right)^{-3}-2^{1-2 w}}{4}(Z(s, w ; 1,-1)-Z(s, w ;-1,-1)) \\
& \quad+\frac{\left(1-\frac{1}{2^{s}}\right)^{-3}-\left(1+\frac{1}{2^{s}}\right)^{-3}}{4}(Z(s, w ; 1,2)+Z(s, w ; 1,-2)+Z(s, w ;-1,2) \\
& \quad-Z(s, w ;-1,-2))+2^{-3 w}(Z(s, w ; 2,1)+Z(s, w ;-2,1)) .
\end{aligned}
$$

Proof. By definition (1.3), we have

$$
Z(s, w)=\sum_{D \text { fund. disc. }}\left(1-\frac{\chi_{D}(2)}{2^{s}}\right)^{-3} \frac{L^{2}\left(s, \chi_{D}\right)^{3}}{|D|^{w}} .
$$

Now (4.2) follows easily from a case-by-case study for the sums over fundamental discriminants $D$ with given sign and given congruence class modulo 8 .

Proposition 6. Let $a, b \in \mathbb{Z}$ with $a b= \pm 1, \pm 2$, and assume that the conditions in Theorem 1 are satisfied. Then

$$
\begin{aligned}
\operatorname{Res}_{w=\frac{3}{4}} Z\left(\frac{1}{2}, w ; a, b\right) & =\sum_{\substack{r=1 \\
2 \nmid r}}^{\infty} \mu(r) \sum_{\ell \mid r} \mu(\ell) \sum_{\delta \mid \ell} \frac{\chi_{b}(\delta) \zeta_{\frac{\ell}{\delta}}(1)^{3}}{\delta^{\frac{3}{4}}} \sum_{m \mid\left(\frac{\ell}{\delta}\right)^{3}} \frac{\tau_{3}\left(m ; \frac{\ell}{\delta}\right) \chi_{a \delta}\left(m_{0}\right)}{m^{\frac{1}{2}}} \\
& \times \sum_{\epsilon= \pm 1} \frac{1+\epsilon \chi-1\left(m_{0}\right)}{2} \operatorname{Res}_{\substack{w=\frac{3}{4} \\
Z_{2 \ell}}}\left(\frac{1}{2}, w ; a \delta, \epsilon b m_{0}\right) .
\end{aligned}
$$

Proof. By our assumption (1.6) in Theorem 1, we have

$$
\operatorname{Res}_{w=\frac{3}{4}} Z\left(\frac{1}{2}, w ; a, b\right)=\sum_{\substack{r=1 \\ 2 \nmid r}}^{\infty} \mu(r) \operatorname{Res}_{w=\frac{3}{4}} Z\left(\frac{1}{2}, w ; a, b ; r\right) .
$$

Furthermore, we have [4]

$$
\begin{aligned}
Z(s, w ; a, b ; r) & =\sum_{\ell \mid r} \mu(\ell) \zeta_{\ell}(2 s)^{3} \sum_{\delta \mid \ell} \frac{\chi_{b}(\delta)}{\delta^{w} \zeta_{\delta}(2 s)^{3}} \sum_{m \mid\left(\frac{\ell}{\delta}\right)^{3}} \frac{\tau_{3}\left(m ; \frac{\ell}{\delta}\right) \chi_{a \delta}\left(m_{0}\right)}{m^{s}} \\
& \times \sum_{\epsilon= \pm 1} \frac{1+\epsilon \chi_{-1}\left(m_{0}\right)}{2} Z_{2 \ell}\left(s, w ; a \delta, \epsilon b m_{0}\right) .
\end{aligned}
$$

Combining this with (4.4) gives the proposition.

Finally, we are ready to explicitly compute the residue of $Z\left(\frac{1}{2}, w\right)$ at $w=\frac{3}{4}$. 
Proof of Theorem 1. The decomposition (4.2) readily gives the meromorphic continuation for $Z\left(\frac{1}{2}, w\right)$ up to $\Re w>\sigma_{0}$, so we focus on the computation of its residue at $w=\frac{3}{4}$.

Theoretically, we can directly compute this residue via Proposition 4 by taking $s=\frac{1}{2}$ there and then applying the sieving equalities (4.2), (4.3). To simplify this process, we observe that many terms given in Proposition 4 contribute trivially to the residue of $Z\left(\frac{1}{2}, w\right)$ at $w=\frac{3}{4}$. More precisely, by Propositions 4 and 6 , the residue of $Z\left(\frac{1}{2}, w ; a, b\right)$, and so that of $Z\left(\frac{1}{2}, w\right)$, at $w=\frac{3}{4}$ is a linear combination of terms of form

$$
\begin{aligned}
R\left(\chi, \chi^{\prime}, \chi^{\prime \prime}\right) & =\lim _{\epsilon \rightarrow 0} \sum_{\substack{r=1 \\
2 \nmid r}}^{\infty} \frac{\mu(r)}{r^{\epsilon}} \sum_{\ell \mid r} \frac{\mu(\ell) \zeta_{\ell}(1)^{2}}{\zeta_{\ell}\left(\frac{1}{2}\right)^{4}} \sum_{\delta \mid \ell} \frac{\chi(\delta)}{\delta^{\frac{3}{4}} \zeta_{\delta}(1)^{3}} \sum_{m \mid\left(\frac{\ell}{\delta}\right)^{3}} \frac{\tau_{3}\left(m ; \frac{\ell}{\delta}\right) \chi^{\prime}(m)}{m^{\frac{1}{2}} m_{0}^{\frac{1}{4}}} \\
& \times \zeta_{\frac{\ell}{m_{0}}}\left(\frac{1}{2}\right) E_{\ell}\left(\frac{3}{4} ; m_{0}\right) A_{\frac{\ell}{m_{0}}}\left(\frac{1}{2}, \chi^{\prime \prime} ; \delta\right),
\end{aligned}
$$

where $\chi, \chi^{\prime}, \chi^{\prime \prime}$ are some quadratic characters of conductors dividing 8 , and the factor $\frac{1}{r^{\epsilon}}$ is inserted as the convergence factor. Now it is easy to see that

$$
\begin{aligned}
R\left(\chi, \chi^{\prime}, \chi^{\prime \prime}\right) & =\lim _{\epsilon \rightarrow 0} \prod_{p \neq 2}\left(1+\frac{9 \chi^{\prime}(p)+\chi^{\prime \prime}(p)+\chi(p) \chi^{\prime \prime}(p)-11}{p^{1+\epsilon}}+O\left(\frac{1}{p^{\frac{3}{2}}}\right)\right) \\
& =c \lim _{\epsilon \rightarrow 0} \frac{L\left(1+\epsilon, \chi^{\prime}\right)^{9} L\left(1+\epsilon, \chi^{\prime \prime}\right) L\left(1+\epsilon, \chi \chi^{\prime \prime}\right)}{\zeta(1+\epsilon)^{11}},
\end{aligned}
$$

where $c>0$ is some constant. Hence $R\left(\chi, \chi^{\prime}, \chi^{\prime \prime}\right)=0$ unless $\chi=\chi^{\prime}=\chi^{\prime \prime}=1$. In fact, the constant $P$ defined in $(1.9)$ is just $\frac{\zeta\left(\frac{1}{2}\right)^{7}}{\zeta_{2}\left(\frac{1}{2}\right)^{3}} R(1,1,1)$. With this at hand, we can apply Propositions 4 and 6 to compute the residue of $Z\left(\frac{1}{2}, w ; a, b\right)$, safely discarding those terms which will introduce nontrivial characters to the summation. A direct computation, combined with (4.2), shows that

$$
\operatorname{Res}_{w=\frac{3}{4}} Z\left(\frac{1}{2}, w\right)=\frac{223 \sqrt{2}-253}{256} P\left(\frac{\Gamma_{\mathbb{R}}\left(\frac{1}{4}\right)^{4}}{\Gamma_{\mathbb{R}}\left(\frac{3}{4}\right)^{4}}+\frac{\Gamma_{\mathbb{R}}\left(\frac{1}{4}\right) \Gamma_{\mathbb{R}}\left(\frac{5}{4}\right)^{3}}{\Gamma_{\mathbb{R}}\left(\frac{3}{4}\right) \Gamma_{\mathbb{R}}\left(\frac{7}{4}\right)^{3}}\right) \approx-0.162 .
$$

This completes the proof.

\section{Proof of Theorem 2}

Finally, we are ready to prove our Theorem 2.

Proof of Theorem 2. By [4], we see that $Z\left(\frac{1}{2}, w\right) \ll 1$ if $\Re w>1$, so

$$
\sum_{\substack{|D| \leq x \\ \text { fund. disc. }}} L\left(\frac{1}{2}, \chi_{D}\right)^{3}=\frac{1}{2 \pi i} \int_{1+\epsilon-i T}^{1+\epsilon+i T} \frac{Z\left(\frac{1}{2}, w\right) x^{w}}{w} \mathrm{~d} w+O\left(\frac{x^{1+\epsilon}}{T}\right) .
$$

Now shift the integration line from $\Re w=1+\epsilon$ to $\Re w=\sigma_{0}+\epsilon$. It is well-known [4] that $Z\left(\frac{1}{2}, w\right)$ has a pole at $w=1$ of order 7 , and we have shown that it also 
has a simple pole at $w=\frac{3}{4}$, so our growth condition (1.10) gives

$$
\sum_{|D| \leq x} L\left(\frac{1}{2}, \chi_{D}\right)^{3}
$$

$D$ fund. disc.

$$
=x R_{3}(\log x)+\frac{4 x^{\frac{3}{4}}}{3} \operatorname{Res}_{w=\frac{3}{4}} Z\left(\frac{1}{2}, w\right)+O\left(T^{r+\epsilon} x^{\sigma_{0}+\epsilon}+\frac{x^{1+\epsilon}}{T}\right) .
$$

where $R_{3}$ is a polynomial of degree 6 . Now if we take $T=x^{\frac{1-\sigma_{0}}{r+1}}$, then this gives

$$
\sum_{\substack{|D| \leq x \\ \text { fund. disc. }}} L\left(\frac{1}{2}, \chi_{D}\right)^{3}=x R_{3}(\log x)+b x^{\frac{3}{4}}+O\left(x^{\frac{r+\sigma_{0}}{r+1}+\epsilon}\right),
$$

where

$$
b=\frac{4}{3} \operatorname{Res}_{w=\frac{3}{4}} Z\left(\frac{1}{2}, w\right) \approx-0.215 .
$$

Note that our assumption on $r$ guarantees $\frac{r+\sigma_{0}}{r+1}<\frac{3}{4}$. This completes the proof of the theorem.

\section{Acknowledgements}

The author would like to thank Dorian Goldfeld for his guidance and support throughout this work, and Adrian Diaconu for many inspiring discussions and suggestions. He would also like to thank Solomon Friedberg, Jeffrey Hoffstein, Stephen Miller and Michael Rubinstein for their helpful comments on this work.

\section{References}

[1] D. Bump, S. Friedberg, J. Hoffstein, Sums of twisted GL(3) automorphic L-functions, Contributions to Automorphic Forms, Geometry and Arithmetic (H. Hida, D. Ramakrishnan and F. Shahidi, eds.), Johns Hopkins Univ. Press, Baltimore, MD, 2004, 131-162.

[2] J. Conrey, D. Farmer, J. Keating, M. Rubinstein, N. Snaith, Integral moments of Lfunctions, math.NT/0206018.

[3] B. Conrey, H. Iwaniec, The cubic moment of central values of automorphic L-functions, Ann. of Math. (2) 151 (2000), 1175-1216.

[4] A. Diaconu, Adrian, D. Goldfeld, J. Hoffstein, Multiple Dirichlet series and moments of zeta and L-functions, Compositio Math., 139 (2003), 297-360.

[5] D. Goldfeld, J. Hoffstein, Eisenstein series of $\frac{1}{2}$-integral weight and the mean value of real Dirichlet L-series, Invent. Math. 80 (1985), 185-208.

[6] D. R. Heath-Brown, A mean value estimate for real character sums, Acta Arith. 72 (1995), 235-275.

[7] M. Jutila, On the mean value of $L\left(\frac{1}{2}, \chi\right)$ for real characters, Analysis 1 (1981), 149-161.

[8] N. Katz, P. Sarnak, Random matrices, Frobenius eigenvalues, and monodromy, American Mathematical Society Colloquium Publications, 45. American Mathematical Society, Providence, RI, 1999.

[9] J. Keating, N. Snaith, Random matrix theory and L-functions at $s=\frac{1}{2}$, Comm. Math. Phys. 214 (2000), 91-110. 
[10] M. Rubinstein, Numerical data on the moments of quadratic Dirichlet L-functions, http://pmmac03.math.uwaterloo.ca/ ${ }^{2}$ mrubinst/L_function_public/MOMENTS/ DIRICHLET/.

[11] G. Shimura, On modular forms of half integral weight, Ann. of Math. (2) 97 (1973), 440-481.

[12] K. Soundararajan, Nonvanishing of quadratic Dirichlet L-functions at $s=\frac{1}{2}$, Ann. of Math. (2) 152 (2000), 447-488.

Department of Mathematics, Johns Hopkins University, Baltimore, MD 21218, U.S.A.

E-mail address: qzhang@math.jhu.edu 